\title{
Cell-Culture Adaptation of H3N2 Influenza Virus Impacts Acid Stability and Reduces Airborne Transmission in Ferret Model
}

\author{
Valerie Le Sage ${ }^{1}$, Karen A. Kormuth ${ }^{1,+}{ }^{\text {, Eric Nturibi }}{ }^{1}$, Juhye M. Lee ${ }^{2,3}$, Sheila A. Frizzell ${ }^{4}$, \\ Michael M. Myerburg ${ }^{4}$, Jesse D. Bloom ${ }^{2,3,5}$ (D) and Seema S. Lakdawala ${ }^{1,6, *}$ \\ 1 Department of Microbiology and Molecular Genetics, University of Pittsburgh School of Medicine, \\ Pittsburg, PA 15219, USA; valerie.lesage@pitt.edu (V.L.S.); kkormuth@bethanywv.edu (K.A.K.); \\ nturibi.eric@medstudent.pitt.edu (E.N.) \\ 2 Division of Basic Sciences and Computational Biology Program, Fred Hutchinson Cancer Research Center \\ Seattle, WA 98109, USA; juhyelee@uw.edu (J.M.L.); jbloom@fredhutch.org (J.D.B.) \\ 3 Department of Genome Sciences, University of Washington, Seattle, WA 98195, USA \\ 4 Department of Medicine, Division of Pulmonary, Allergy, and Critical Care Medicine, \\ University of Pittsburgh School of Medicine, Pittsburgh, PA 15219, USA; samfrizzell@icloud.com (S.A.F.); \\ myerburgm@upmc.edu (M.M.M.) \\ 5 Howard Hughes Medical Institute, Seattle, WA 98103, USA \\ 6 Center for Vaccine Research, University of Pittsburgh School of Medicine, Pittsburgh, PA 15219, USA \\ * Correspondence: Lakdawala@pitt.edu \\ check for \\ updates \\ + Current address: Bethany College, Bethany, WV 26032, USA.
}

Citation: Le Sage, V.; Kormuth, K.A.; Nturibi, E.; Lee, J.M.; Frizzell, S.A.; Myerburg, M.M.; Bloom, J.D.; Lakdawala, S.S. Cell-Culture Adaptation of H3N2 Influenza Virus Impacts Acid Stability and Reduces Airborne Transmission in Ferret Model. Viruses 2021, 13, 719. https://doi.org/10.3390/ v13050719

Academic Editors: Jessica A. Belser, Joanna A. Pulit-Penaloza and Xiangjie Sun

Received: 9 March 2021

Accepted: 14 April 2021

Published: 21 April 2021

Publisher's Note: MDPI stays neutral with regard to jurisdictional claims in published maps and institutional affiliations.

Copyright: (c) 2021 by the authors. Licensee MDPI, Basel, Switzerland. This article is an open access article distributed under the terms and conditions of the Creative Commons Attribution (CC BY) license (https:// creativecommons.org/licenses/by/ $4.0 /)$.
Abstract: Airborne transmission of seasonal and pandemic influenza viruses is the reason for their epidemiological success and public health burden in humans. Efficient airborne transmission of the $\mathrm{H} 1 \mathrm{~N} 1$ influenza virus relies on the receptor specificity and $\mathrm{pH}$ of fusion of the surface glycoprotein hemagglutinin (HA). In this study, we examined the role of HA pH of fusion on transmissibility of a cell-culture-adapted H3N2 virus. Mutations in the HA head at positions 78 and 212 of A/Perth/16/2009 (H3N2), which were selected after cell culture adaptation, decreased the acid stability of the virus from $\mathrm{pH} 5.5$ (WT) to $\mathrm{pH} 5.8$ (mutant). In addition, the mutant H3N2 virus replicated to higher titers in cell culture but had reduced airborne transmission in the ferret model. These data demonstrate that, like H1N1 HA, the $\mathrm{pH}$ of fusion for H3N2 HA is a determinant of efficient airborne transmission. Surprisingly, noncoding regions of the NA segment can impact the $\mathrm{pH}$ of fusion of mutant viruses. Taken together, our data confirm that HA acid stability is an important characteristic of epidemiologically successful human influenza viruses and is influenced by HA/NA balance.

Keywords: influenza virus; transmission; $\mathrm{pH}$; hemagglutinin

\section{Introduction}

Influenza A viruses cause acute respiratory disease in mammals and birds, whereas aquatic avian species and bats act as zoonotic reservoirs. Host tropism is determined by a combination of viral proteins and host factors which allow for efficient replication and transmission within a given species. Influenza virus particles have two major surface glycoproteins, hemagglutinin (HA) and neuraminidase (NA). The trimeric HA protein binds sialic acids on cell-membrane-bound proteins and is a major determinant of host tropism. Avian HA proteins preferentially bind to an $\alpha 2,3$-linked sialic acid, whereas human viruses have an $\alpha 2,6$-linked sialic acid preference [1-3]. HA is functionally balanced by the tetrameric receptor-destroying NA. At later stages of infection, NA plays a major role in removing sialic acids from host-cell receptors, as well as from newly synthesized HA and NA on nascent virions, which are sialylated during transport through the endoplasmic reticulum and Golgi apparatus [4,5]. Sialic acid removal by NA prevents virion aggregation and promotes spread to new target cells [5]. The functional balance between HA and 
NA is important to the maintenance of viral fitness, as NA needs to be active enough to disaggregate virions upon release but not so active that HA receptor attachment is decreased [6].

Proteolytic cleavage of the HA0 precursor by host proteases produces HA1 and HA2 subunits to reveal the fusion peptide, which is necessary for membrane fusion $[7,8]$. Once bound to sialic acids, influenza viruses are internalized via receptor-mediated endocytosis and HA mediates escape from early endosomes in a $\mathrm{pH}$-dependent manner. At a fixed $\mathrm{pH}$, HA undergoes an irreversible conformational change, which facilitates fusion of the viral and cellular membranes and the consequent release viral genomes into the host cytoplasm [9]. The pH stability of viral HA varies by host origin. The HA protein from circulating human influenza viruses undergoes a confirmation change at pH 5.0-5.4 [10-12]. In contrast, avian and swine influenza viruses are less acid stable, and their HA proteins undergo a conformational change at a $\mathrm{pH}>5.5$ [12-19]. Thus, $\mathrm{HA} \mathrm{pH}$ of fusion is an important factor in host adaptation and transmissibility [12,20-22].

The importance of HA pH of fusion in the cell adaptation and transmission of H3N2 viruses is unclear. In this study, we demonstrated that cell adaptation of a seasonal 2009 H3N2 virus (A/Perth/16/2009) resulted in HA mutations (G78D and T212I) [23] that decreased HA acid stability. The adapted H3N2 virus with these two HA mutations (herein referred to as the 'rPerth mutant') exhibits enhanced replication in MDCK cells as compared to the wild-type virus (herein referred to as 'rPerth $\mathrm{WT}^{\prime}$ '). However, no growth difference was observed in more physiologically relevant adenocarcinoma human alveolar basal epithelial (A549) and human bronchial epithelial (HBE) cells. In ferrets, the rPerth mutant replicated similarly to rPerth WT, but displayed reduced efficiency of transmission via respiratory droplets between ferrets. Our observations suggest that, similarly to H1N1 viruses, H3N2 viruses with a higher $\mathrm{pH}$ of fusion have decreased airborne transmission, confirming the importance of this phenotype for transmission fitness.

\section{Material and Methods}

\subsection{Cells and Viruses}

MDCK epithelial cells (ATCC, CCL-34) were maintained in Eagle's minimal essential medium (MEM) supplemented with 10\% fetal bovine serum (FBS), penicillin/streptomycin, and L-glutamine. A549 and 293T cells (ATCC, CCL-185 and CRL-11268) were maintained in Dulbecco's MEM (DMEM) supplemented with 10\% FBS, penicillin/streptomycin, and L-glutamine. Primary HBE cells derived from human lung tissue were differentiated and cultured at an air-liquid interface using a protocol approved by the relevant institutional review board [24]. The H3N2 viruses A/Panama/2007/2009, A/Perth/16/2009, A/Wyoming/3/2003, and A/Wisconsin/67/2005 were a generous gift from Dr Zhiping Ye (Center for Biologics Evaluation and Research, FDA, Silver Spring, MD, USA). A/Minnesota/11/2010 H3N2v and sw/OK/011506/2007 H3N2 viruses were a kind gift from Dr Kanta Subbarao (Doherty Institute, Melbourne, Australia). Influenza virus titers were determined by TCID $_{50}$ assay on MDCK cells according to the method of Reed and Muench [25].

\subsection{Plasmid-Based Reverse Genetics}

The viruses used in this study were generated using bidirectional reverse-genetics plasmids based on pHW2000 [26]. The plasmids were cloned from cDNA reverse transcribed from the A/Perth/16/2009 (H3N2) virus stock described in the subsection immediately above, and were named pHW-Perth09-PB2-SL, pHW-Perth09-PB1-SL, pHW-Perth09-PA-SL, pHW-Perth09-HA-SL, pHW-Perth09-NP, pHW-Perth09-NA, pHW-Perth09-M, and pHWPerth09-NS1. The noncoding regions of the pHW-Perth09-HA-SL and pHW-Perth09-NA-SL exactly match the noncoding regions of the comparable vRNAs deposited in Genbank by the WHO Collaborating Centre for influenza research in Australia (Genbank GQ293081 and GQ293082), except for a single A nucleotide inserted after the 21st nucleotide in the noncoding region at the $5^{\prime}$ end of the NA vRNA. We also used the variant HA and NA plasmids 
described in Reference [23] as pHW-Perth09-HA-G78D-T212I and pHW-Perth09-NA. These variant plasmids differ in two ways from the initial set of eight: they have noncoding regions from the lab-adapted X-31 strain, and the HA has two amino-acid mutations that were selected after passaging in cell culture (see Reference [23] for details). The virus termed 'rPerth $\mathrm{WT}^{\prime}$ was generated from the pHW-Perth09-*-SL series of plasmids, whereas the virus termed 'rPerth mutant' used the pHW-Perth09-HA-G78D-T212I and pHW-Perth09-NA plasmids and the other six genes from the pHW-Perth09-*-SL series. The sequences of all plasmids are provided on FigShare at https:/ / figshare.com/projects/Cell_Culture_Adaptation_of_H3N2 _Influenza_Virus_Impacts_Acid_Stability_and_Reduces_Airborne_Transmission_in_Ferrets/ 112005(accessed on 21 April 2021).

Recombinant viruses were generated using the eight reverse-genetics plasmids transfected into 293T cells, using TransIT-LT1 transfection reagent (Mirus Bio LLC, Madison, WI, USA) in accordance with the manufacturer's protocol. After 24 and $48 \mathrm{~h}$, 293T cell supernatants were used to infect MDCK cells to rescue a CP1 stock of virus.

\subsection{Replication Kinetics}

A549 and MDCK cells were infected with the indicated virus at a multiplicity of infection (MOI) of 0.01 or 1.0 (calculated on the basis of the TCID ${ }_{50}$ ) in MEM containing $2 \%$ L-glutamine and supplemented with $1 \mu \mathrm{g} / \mathrm{mL}$ L-(tosylamido-2-phenyl) ethyl chloromethyl ketone (TPCK)-treated trypsin (Worthington Biochemical, Lakewood, NJ, USA). Virus was allowed to adsorb for $1 \mathrm{~h}$ before the inoculum was removed and replaced with fresh medium. Supernatants were collected at the indicated time points and titered by TCID 50 .

Three different HBE patient cultures were used: HBE0176, HBE0256, and HBE259. The apical surface of the HBE cells was washed in phosphate-buffered saline (PBS), and $10^{3}$ TCID $_{50}$ of virus was added per $100 \mu \mathrm{L}$ of HBE growth medium. After a $1 \mathrm{~h}$ incubation at room temperature, the inoculum was removed and the apical surface was washed three times with PBS. At the indicated time points, $150 \mu \mathrm{L}$ of HBE medium was added to the apical surface for $10 \mathrm{~min}$ to capture released virus particles. The experiment was performed in triplicate in three different patient cell lines.

\subsection{Animal Ethics Statement}

Ferret experiments were conducted in a BSL2 facility at the University of Pittsburgh in compliance with the guidelines of the Institutional Animal Care and Use Committee (approved protocols \#16077170 and \#19075697). Animals were sedated with isoflurane following approved methods for all nasal washes and survival blood draws. Ketamine and xylazine were used for sedation for all terminal procedures, followed by cardiac administration of euthanasia solution. Approved University of Pittsburgh Division of Laboratory Animal Resources (DLAR) staff administered euthanasia at time of sacrifice.

\subsection{Ferret Screening}

Five to six month old male ferrets were purchased from Triple F Farms (Sayre, PA, USA). All ferrets were screened by hemagglutinin inhibition assay for antibodies against circulating influenza A and B viruses, as described in Reference [27], using the following antigens obtained through the International Reagent Resource, Influenza Division, WHO Collaborating Center for Surveillance, Epidemiology and Control of Influenza, Centers for Disease Control and Prevention, Atlanta, GA, USA: 2018-2019 WHO Antigen, Influenza A (H3) Control Antigen (A/Singapore/INFIMH-16-0019/2016), BPLInactivated, FR-1606; 2014-2015 WHO Antigen, Influenza A (H1N1)pdm09 Control Antigen (A/California/07/2009 NYMC X-179A), BPL-Inactivated, FR-1184; 2018-2019 WHO Antigen, Influenza B Control Antigen, Victoria Lineage (B/Colorado/06/2017), BPLInactivated, FR-1607; 2015-2016 WHO Antigen, Influenza B Control Antigen, Yamagata Lineage (B/Phuket/3073/2013), BPL-Inactivated, FR-1403. 


\subsection{Transmission Studies}

Our transmission caging setup was a modified Allentown ferret and rabbit bioisolator cage similar to those used in our previous studies, described in References [27-29]. For each study, three ferrets were anesthetized with isoflurane and inoculated intranasally with $10^{6} \mathrm{TCID}_{50} / 500 \mu \mathrm{L}$ of A/Perth/16/2009 WT or mutant virus to act as donor animals. Twenty-four hours later, a recipient ferret was placed into the cage but separated from the donor animal by two staggered perforated metal plates welded together one inch apart. Recipients were exposed for 14 days. Nasal washes were collected from each donor and recipient every other day for 14 days. To prevent accidental contact or fomite transmission by investigators, the recipient ferrets were handled first and extensive cleaning of gloves, chambers, biosafety cabinet, and temperature monitoring wand was performed between each recipient and donor animal and between each pair of animals. Sera were collected from donor and recipient ferrets upon completion of experiments to confirm seroconversion. To ensure no accidental contact or fomite transmission during husbandry procedures, recipient animal sections of the cage were cleaned prior to the donor sides, with three lab personnel participating in each husbandry event to ensure that a clean pair of hands handled bedding and food changes. One cage was done at a time, with a $10 \mathrm{~min}$ wait time between cages to remove contaminated air prior to moving on to the next cage. Fresh scrapers, gloves, and sleeve covers were used for each subsequent cage cleaning. Clinical symptoms such as weight loss and temperature were recorded during each nasal wash procedure and other symptoms such as sneezing, coughing, lethargy or nasal discharge were noted during any handling events. Once animals reached $10 \%$ weight loss, their feed was supplemented with A/D diet twice a day to entice eating. A summary of clinical symptoms are provided in Table 1.

Table 1. Airborne transmission of rPerth WT and mutant viruses.

\begin{tabular}{|c|c|c|c|c|c|c|c|}
\hline Virus & Exposure Time & Status & Transmission Efficiency & Temperature Increase * & Weight loss* & $\begin{array}{c}\mathrm{H} 3 \mathrm{~N} 2 \\
\underset{\text { Microneutralization }}{\text { Titers }}{ }^{\wedge}\end{array}$ & HAI Titers ^ \\
\hline \multirow{4}{*}{$\begin{array}{l}\text { rPerth WT } \\
\text { rPerth mutant }\end{array}$} & 14 days & INF & \multirow{3}{*}{$3 / 3$} & $0 / 3$ & $0 / 3$ & $2560,250,2560$ & $2560,250,2560$ \\
\hline & \multirow{3}{*}{14 days } & Naïve & & $1 / 3$ & $0 / 3$ & $1280,2560,1280$ & $1280,2560,1280$ \\
\hline & & INF & & $1 / 3$ & $0 / 3$ & $2260,1010,2260$ & $1280,1280,690$ \\
\hline & & Naïve & $1 / 3$ & $1 / 3$ & $0 / 3$ & $<20,<20,1010$ & $<10,<10,2560$ \\
\hline
\end{tabular}

* Temperature increase is defined as $>1.5 \mathrm{deg}$ from day 0 . Weight loss determined as $>10 \%$ of day $0 .{ }^{\wedge}$ Antibody titers of day 14 are presented. All day 0 sera had a titer $<20 .{ }^{\wedge}$ Antibody titers of day 14 are presented. All day 0 sera had a titer $<10$.

\subsection{Serology Assays}

Analysis of neutralizing antibodies from ferret sera was performed as previously described [27]. Briefly, the microneutralization assay was performed using $10^{3.3} \mathrm{TCID}_{50}$ of either H3N2 virus incubated with 2-fold serial dilutions of heat-inactivated ferret serum. The neutralizing titer was defined as the reciprocal of the highest dilution of serum required to completely neutralize the infectivity of $10^{3.3} \mathrm{TCID}_{50}$ of virus on MDCK cells. The concentration of antibody required to neutralize $100 \mathrm{TCID}_{50}$ of virus was calculated based on the neutralizing titer dilution divided by the initial dilution factor, multiplied by the antibody concentration.

For hemagglutinin inhibition (HAI) assays, serum samples were pretreated with receptor-destroying enzyme (Seiken) followed by hemadsorption. Briefly, sera were serially diluted 2-fold in a V-bottom plate and mixed with four agglutinating units of virus for $15 \mathrm{~min}$. An equal volume of $0.5 \%$ turkey erythrocytes was gently added to each well and incubated for $30 \mathrm{~min}$ at room temperature. Agglutination was read and HAI titers were expressed as the inverse of the highest dilution that inhibited four agglutinating units of virus. 


\subsection{Tissue Sample Collection}

Euthanized ferrets were dissected aseptically. Collection of respiratory tissues was performed in the following order: entire right middle lung, left cranial lung (a portion equivalent to the right middle lung lobe), one inch of trachea cut lengthwise, entire soft palate, and nasal turbinates. Tissues were harvested as described in Reference [28] and frozen at $-80^{\circ} \mathrm{C}$. Tissue samples were weighed and Leibovitz's L-15 medium (Invitrogen) was added to make a $10 \% w / v$ homogenate. Tissues were dissociated using an OMNI GLH (OMNI International, Kennesaw, GA, USA) homogenizer and cell debris was removed by centrifugation at $1500 \mathrm{rpm}$ for $10 \mathrm{~min}$. Infectious virus was quantified by $\mathrm{TICD}_{50}$ using the endpoint method [25].

\subsection{In Vitro HA $p H$ Inactivation Assay}

Virus stocks were incubated in PBS adjusted to the indicated $\mathrm{pH}$ values for $1 \mathrm{~h}$ at $37^{\circ} \mathrm{C}$. The infectious titer of the virus after incubation was determined on MDCK cells using the $\mathrm{TCID}_{50}$ endpoint titration method [25]. The $\mathrm{pH}$ that reduced the titer by $50 \%$ $\left(\mathrm{EC}_{50}\right)$ was calculated by regression analysis of the dose-response curves. Each experiment was performed in triplicate in at least two independent biological replicates.

\section{Results}

3.1. Viruses with Cell-Culture-Adaptive HA Mutations Replicated Better in MDCK Cells

A previous study by members of our team serially passaged a 6:2 reassortant virus of H3N2 virus with HA and NA genes from A/Perth/16/2009 and internal WSN segments six times in MDCK-SIAT1 cells expressing TMPRSS2. Two mutations in the HA segment, G78D and T212I (H3 numbering), arose after serial passages [23]. To compare the replication kinetics of viruses carrying these cell-culture-adapted mutations, reverse genetics was used to generate both wild-type (WT) and HA mutant virus strains with A/Perth/16/2009 internal gene segments (Figure 1A). The genes for all of these viruses were cloned from a stock of A/Perth/16/2009 (H3N2) virus; however, the HA and NA genes differed between the WT and mutant viruses. Recombinant A/Perth/16/2009 WT (rPerth WT) was generated from reverse-genetic plasmids with native $5^{\prime}$ and $3^{\prime}$ untranslated regions (UTRs) for the HA and NA segments (Figure 1A and Supplementary File 1). However, the mutant strain (rPerth mutant) was generated from plasmids in which the HA and NA were cloned into reverse-genetic plasmids with the noncoding regions from the lab-adapted H3N2 reassortant strain $\mathrm{X}-31$, with the HA also containing the two lab-adaptation mutations G78D and T212I (Figure 1A and Supplementary File 1).

Replication kinetics of rPerth WT and rPerth mutant viruses were examined in MDCK and A549 cells. Each cell line was infected with rPerth WT or rPerth mutant virus at a multiplicity of infection (MOI) of 1.0 or 0.01 , and the viral titers in infected cell supernatants were collected at the indicated time points. At the higher MOI in MDCK cells, the rPerth mutant had titers that were $10 \times$ greater at 16,24 , and $48 \mathrm{~h}$ post-infection (hpi) as compared to those of the rPerth WT (Figure 1B). Similarly, the rPerth mutant grew better than rPerth WT at 24 and 48 hpi under a low MOI (Figure 1C). However, in A549 cells, rPerth mutant replicated better than rPerth WT at 48 hpi only at an MOI of 1.0, and to similar titers under all other conditions (Figure 1D,E).

Primary HBE cell cultures differentiated at an air-liquid interface mimic the lumen of the human airway because they produce mucus and are permissive to influenza viruses $[24,30]$. The replication capacity of the rPerth mutant was characterized in three different patient-derived HBE cultures. HBE cells were infected with a low MOI of either rPerth WT or the mutant strain, and released virions were collected at the indicated times post-infection. Regardless of the patient culture, no significant difference in replication between rPerth WT and mutant strains was observed over time (Figure 2). Taken together, these data demonstrate that serial passage of the rPerth mutant on MDCK cells increased replication capacity in MDCK cells, but not in other cell lines or primary airway cultures. 
A

rPerth WT

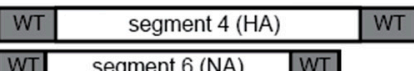

(PB2)

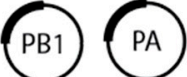

rPerth mutant

\begin{tabular}{|l|l|l|}
\hline $\mathrm{X}-31$ & segment $4(\mathrm{HA}), \mathrm{G} 78 \mathrm{D} / \mathrm{T} 212 \mathrm{I}$ & $\mathrm{X}-31$ \\
\hline $\mathrm{X}-31$
\end{tabular}

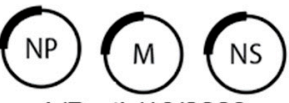

A/Perth/16/2009

internals
B

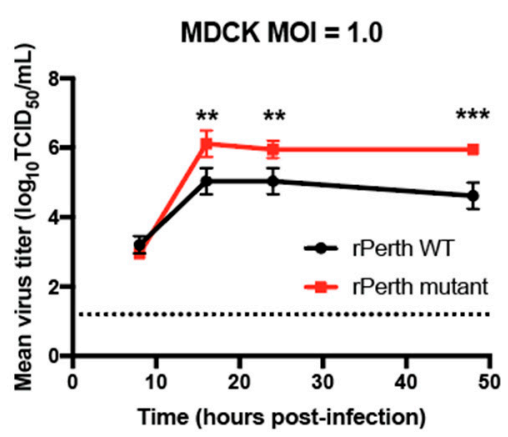

D

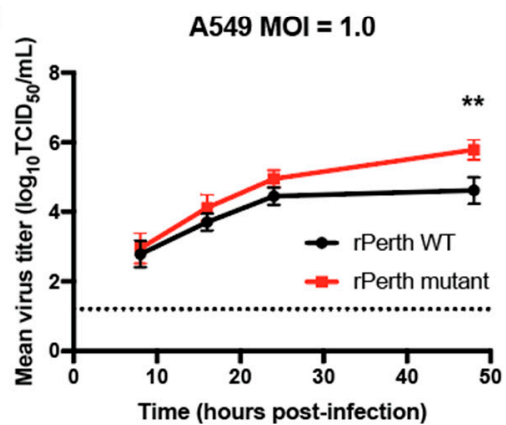

C

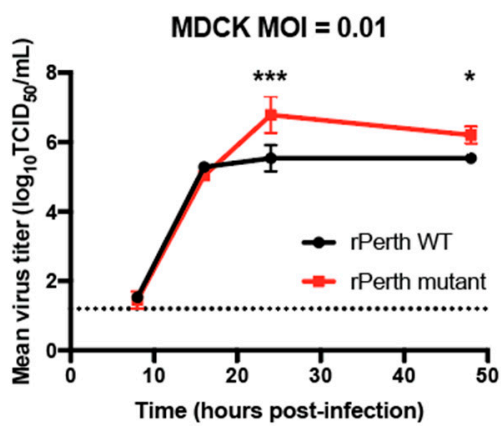

E

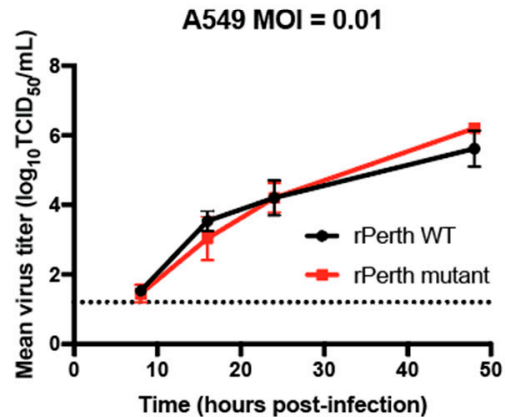

Figure 1. In vitro replication of cell-culture-adapted H3N2 virus. (A) Schematic representation of recombinant (r) A/Perth/16/2009 wild-type (WT) and mutant virus constructs. MDCK (B,C) and A549 (D,E) cells were infected with rPerth WT (black circles) and HA mutant (red squares) viruses at MOIs of 1.0 or 0.01 . Cells were infected in triplicate and supernatants were collected at the indicated times. Virus titers were determined on MDCK cells using TCID 50 assays. Graphs are representative of three independent experiments. Two-way ANOVA was used to determine statistical significance $\left({ }^{*} p<0.05,{ }^{* *} p<0.005,{ }^{* *} p<0.0005\right)$. The dashed line denotes the limit of detection for the titration assay.
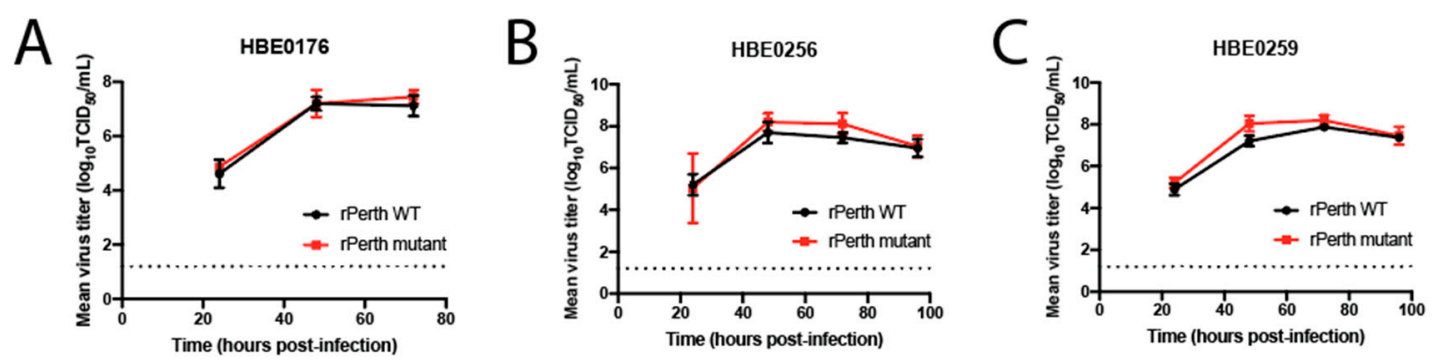

Figure 2. In vitro replication of rPerth WT and HA mutant in human bronchiole epithelial (HBE) cells. Cells from three different patient cell lines, (A) HBE0176, (B) HBE0256, and (C) HBE0259, were infected at $10^{3}$ TCID $_{50}$ per well with rPerth WT (black circles) or rPerth mutant (red squares). The apical supernatant was collected at the indicated time points and virus titers were determined on MDCK cells using TCID $_{50}$ assays. The experiments were performed in triplicate. 


\subsection{The $p H$ of Inactivation rPerth Mutant Was Higher Than That of WT}

Cell-culture adaptation has been associated with a wider range of $\mathrm{pH}$ at which HA fuses with the host endosomal membrane, as determined by syncytia assay, compared to WT viruses [31-33]. Incubation of virions with acidic PBS at different $\mathrm{pH}$ can cause a conformational change in the HA, which results in premature activation of $\mathrm{HA}$ and an irreversible loss of viral infectivity [18]. The $\mathrm{pH}$ of inactivation for rPerth WT and mutant was determined from the infectious titers of virus solutions incubated in $\mathrm{pH}$-adjusted PBS for $1 \mathrm{~h}$ (Figure 3A). The $\mathrm{pH}$ of inactivation acts as a surrogate for the $\mathrm{pH}$ of fusion $[34,35]$ and is expressed as the $\mathrm{EC}_{50}$ from the acid stability assays, which is the $\mathrm{pH}$ that reduces the viral titer by $50 \%$. The $\mathrm{EC}_{50} \mathrm{pH}$ of inactivation for rPerth WT was calculated for each curve and resulted in a $\mathrm{pH}$ of inactivation of 5.57, while that of the rPerth mutant was 5.83 (Figure 3A, black dashed lines). This experiment suggests that MDCK cell-adaptive mutations resulted in a virus with decreased acid stability.
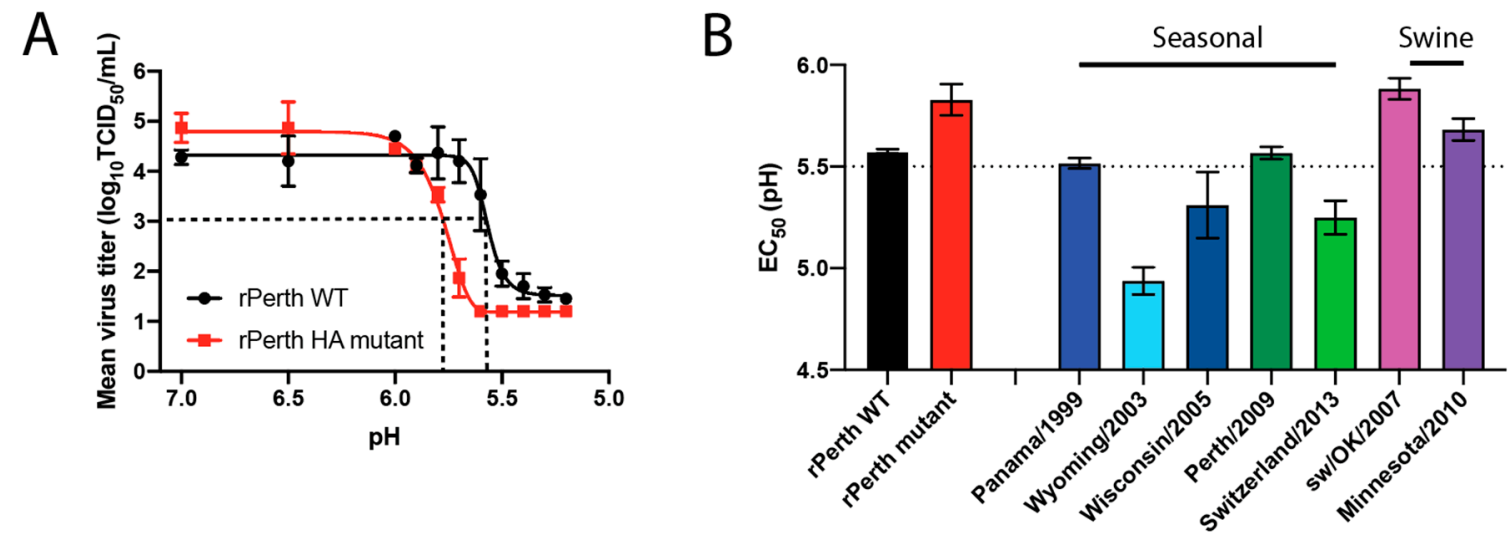

Figure 3. rPerth mutant had decreased HA stability. (A) rPerth WT (black line) and mutant (red line) were treated in $\mathrm{pH}$-adjusted PBS for $1 \mathrm{~h}$ at $37^{\circ} \mathrm{C}$. Remaining virus titers were determined by $\mathrm{TCID}_{50}$ assay. The experiment was performed in triplicate, and representative data are shown. The data were fit with an asymmetric sigmoidal curve to determine the $\mathrm{EC}_{50}$. The limit of detection was $1.2 \log _{10} \mathrm{TCID}_{50} / \mathrm{mL}$. (B) Seasonal and swine H3N2 viruses were incubated in PBS of different $\mathrm{pHs}$ for $1 \mathrm{~h}$ at $37^{\circ} \mathrm{C}$, with experiments performed in triplicate. The remaining virus titers were determined by $\mathrm{TCID}_{50}$ assay and the $\mathrm{EC}_{50}$ values were plotted using regression analysis of the dose-response curve. The reported mean $( \pm \mathrm{SD})$ corresponds to two independent biological replicates, each performed in triplicate.

To examine the acid stability of other seasonal human- or swine-origin H3N2 viruses, we evaluated the $\mathrm{pH}$ of inactivation for a range of contemporary seasonal and swine H3N2 viruses. As expected, the biological A/Perth/16/2009 had the same $\mathrm{pH}$ of inactivation $\left(\mathrm{EC}_{50}=5.57\right)$ as the rPerth WT, while the seasonal human viruses tested ranged in $\mathrm{pH}$ of inactivation from 5.5 to 4.9 (Figure 3B). These data are consistent with human seasonal H1N1 viruses, which have been shown to have a $\mathrm{pH}$ of inactivation $<5.5$ [36]. The two $\mathrm{H} 3 \mathrm{~N} 2$ swine viruses tested had $\mathrm{EC}_{50}$ values that were higher $\left(\mathrm{EC}_{50}=5.88\right.$ and 5.68$)$ and more similar to the rPerth mutant (Figure 3B).

\subsection{Replication of the rPerth Mutant in Ferrets Was Similar to WT}

Based on the replication fitness in the various cell-culture systems and differential $\mathrm{pH}$ of fusion value for these two viruses, we chose to compare the replication capacity in vivo. Ferrets are an established model for the study of influenza pathogenicity and transmissibility because their lung physiology, receptor expression patterns, clinical signs, and transmissibility patterns resemble those of humans [37]. To determine the infectivity of the rPerth mutant in ferrets, three animals were intranasally inoculated with either rPerth WT or rPerth mutant virus. On day 3 post-infection, the ferrets were sacrificed and respiratory tract tissues (nasal turbinates, soft palate, trachea, right middle lung and left cranial lung) were collected. The amount of viral RNA present in the tissue homogenates was assessed by quantitative PCR and infectious virus. Based on viral RNA quantification, 
no differences were observed between the recovery of rPerth WT and mutant viruses in any of respiratory tract tissues collected (Figure 4A). Infectious virus was determined on MDCK cells and was primarily observed in the upper respiratory tract (nasal wash, nasal turbinate, and soft palate) (Figure 4B). No significant difference was observed between infectious virus titers from rPerth-WT- and rPerth-mutant-infected ferrets. The discrepancy in detection of viral RNA and infectious virus may have been due to issues with freezethaw behavior of the homogenates and titration on MDCK cells rather than MDCK-SIAT cells. Taken together, these observations suggest that cell-adapted H3N2 viruses replicate similarly in ferrets, in line with replication data from HBE cells rather than MDCK cells.

A

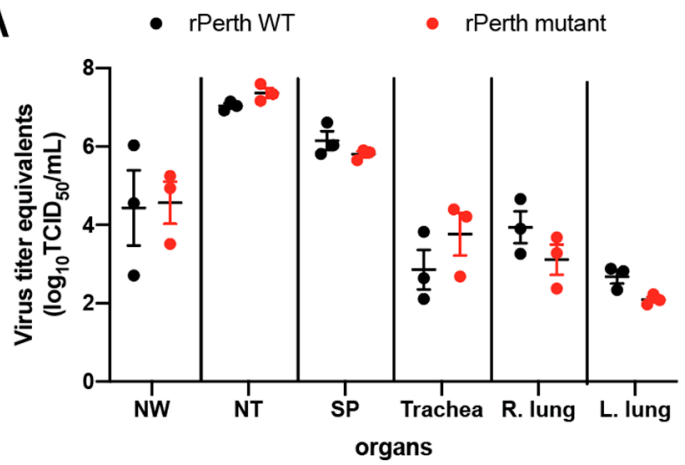

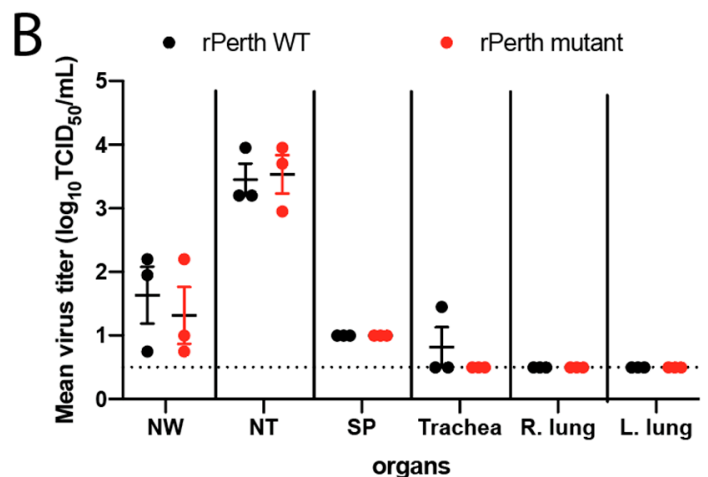

Figure 4. Quantification of H3N2 virus in respiratory tissues of infected ferrets. Ferrets were intranasally infected with $10^{6}$ $\mathrm{TCID}_{50}$ in $0.5 \mathrm{~mL}$ of rPerth WT (black circles) or rPerth mutant (red circles) and were sacrificed on day 3 post-infection. NW—nasal wash, NT_nasal turbinate, SP—soft palate. (A) RNA was isolated from the indicated respiratory tract organ homogenates and qPCR for a region of the M segment was performed to quantify the relative amounts of influenza virus, normalized to RNA isolated from a virus stock with a known titer. (B) Titration of tissue homogenates to quantify infectious viral titers. Each dot represents a single animal and mean line \pm SEM is depicted.

\subsection{H3N2 Mutant Virus with a Higher pH of Fusion Had Reduced Airborne Transmission to Nä̈ve Ferrets}

HA stability is an important factor that impacts transmission efficiency [12,20,21]. To determine whether the $\mathrm{pH}$ stability of $\mathrm{H} 3 \mathrm{~N} 2$ confers an airborne transmission disadvantage and assess the impact of prolonged MDCK cell passage on the transmission efficiency of the rPerth mutant, an airborne transmission study was performed for each virus. Three donor ferrets were infected intranasally with either rPerth WT or rPerth mutant and $24 \mathrm{~h}$ later a naïve recipient ferret was placed in the adjacent cage, with directional air flow from the donor to recipient $[27,29]$. The naïve recipient was exposed for 14 days. Viral titers in nasal washes were collected every other day and seroconversion was determined on day 14 post-infection. rPerth WT transmitted to three out of three recipients, while the transmission efficiency of the rPerth mutant was reduced to one out of three recipients (Table 1). All donors and recipients that shed virus in their nasal washes also seroconverted (Table 1). These results indicate that the cell-adapted rPerth mutant virus has reduced airborne transmission compared to WT.

\subsection{NA-Segment Noncoding Regions Influence the Virus $p H$ of Fusion}

To determine the contribution of the X-31 UTRs to the $\mathrm{pH}$ of inactivation, H3N2 viruses with different combinations of HA and NA (WT versus X-31 UTRs) were generated using reverse genetics. Retention of the rPerth HA X-31 UTRs with the head mutations (G78D and T212I) along with the WT NA segment produced a $\mathrm{pH}$ of inactivation $\left(\mathrm{EC}_{50}=5.56\right)$ which was similar to that of rPerth WT $\left(E_{50}=5.57\right)$ (Figure 5). However, a rPerth virus strain containing the WT HA segment and the X-31 UTR NA segment had a lower $\mathrm{pH}$ of inactivation $\left(\mathrm{EC}_{50}=5.39\right)$ compared to rPerth WT (Figure 5), indicating that X-31 UTR flanked NA segment was necessary but not sufficient to alter the $\mathrm{pH}$ of inactivation. These 
results show that both HA (G78D and T212I) and NA X-31 UTR segments are necessary, but are not sufficient alone to increase the $\mathrm{pH}$ of inactivation for the rPerth virus.

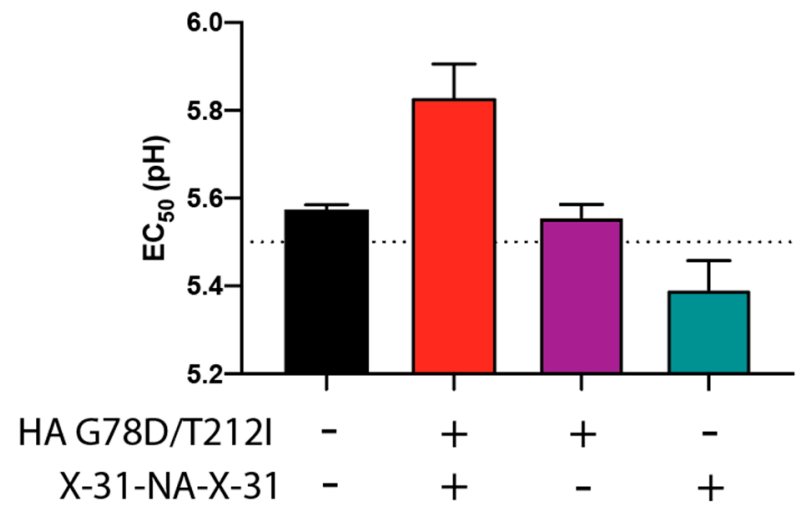

Figure 5. Increased $\mathrm{pH}$ of inactivation requires $\mathrm{X}-31$ UTRs in NA segment and HA mutations. The indicated mutant $\mathrm{H} 3 \mathrm{~N} 2$ viruses were incubated in PBS of different $\mathrm{pHs}$ for $1 \mathrm{~h}$ at $37{ }^{\circ} \mathrm{C}$, with experiments performed in triplicate. The remaining virus titers were determined by $\mathrm{TCID}_{50}$ assay and the $\mathrm{EC}_{50}$ values were plotted using regression analysis of the dose-response curve. The reported means $( \pm \mathrm{SD})$ correspond to two independent experiments performed in three technical replicates.

\section{Discussion}

Cross-species host adaptation is common in influenza viral infections and is the result of selective pressures at the site of replication, cell receptor availability, and compatibility with host transcription and translational machinery. Only H1N1, H2N2, and H3N2 influenza virus subtypes have become established within the human population and transmit person-to-person, while avian $\mathrm{H} 5$ and $\mathrm{H} 7$ influenza viruses will sporadically infect humans without efficient transmission between individuals. Gain-of-function studies with H5N1 influenza viruses have indicated that mutation of HA to confer $\alpha 2,6$-linkage sialic acid binding, loss of glycosylation within the HA head domain, and increasing HA stability improves airborne transmissibility in the ferret model $[20,21]$. Evolution of HA is an important player in interspecies transmission and host range expansion. Mutations that stabilize the H5 HA can enhance replication in the upper respiratory tracts of mice and ferrets $[34,35,38]$, but result in a concomitant decrease in replication, virulence, and transmissibility in the natural avian host $[39,40]$. Conversely, adaptation of human influenza viruses to a murine host required adaptive changes in the HA protein to alter receptor preference and resulted in decreased acid stability from 5.2-5.3 to 5.6-5.8 [41-43].

In this study, we demonstrated that MDCK cell-culture adaptation of an H3N2 influenza virus resulted in a virus with lower acid stability than the WT virus. The HA pH of fusion is a known determinant of host adaptation. Consistent with this, we found that human seasonal viruses have an HA pH of fusion of 4.9-5.5, while swine H3N2 viruses have a $\mathrm{pH}$ of fusion greater than 5.5. Previous work has shown a similar phenotype with human and gamma clade swine H1N1 viruses [36]. Additionally, gamma clade swine H1 viruses replicate to higher titers in MDCK cells [36], which is consistent with our observations of the rPerth mutant. Interestingly, the observation of increased replication capacity was not recapitulated in HBE cells and ferrets, suggesting that MDCK replication capacity is not a strong correlate to results in relevant organoid cultures or in vivo.

HA is an important determinant of influenza virus transmissibility, as it needs to remain stable as it travels in the environment between hosts [12,44]. HA proteins that are stable at acidic $\mathrm{pH}$ have an advantage because they are less prone to being inactivated in their environment [45-47]. However, striking a fine $\mathrm{pH}$ balance is important for an influenza virus, since a replicative advantage is conferred within the host cell to viruses that encode a less acid-stable HA, as this facilitates efficient viral uncoating in endosomes [48]. However, avian virus HA proteins with a higher activation $\mathrm{pH}$ value have some advantages over their more acid-stable human-adapted HA counterparts. During macrophage 
infection, less acid-stable HA proteins release avian viruses earlier from the endosome to escape lysosomal degradation and allow continued replication [49]. Furthermore, a higher membrane fusion $\mathrm{pH}$ has been shown to help avian virus HA proteins avoid detection by the interferon-inducible transmembrane proteins IFITM2 and IFITM3, which restrict virus fusion [50]. Mutations in the head region of the rPerth mutant, which arose during repeated passage in MDCK cells, led to destabilization of HA and raised its activation $\mathrm{pH}$. Similarly, MDCK cell-culture adaption of egg-grown H3N2 X-31 virus caused mutations in or near the fusion peptide, which resulted in higher $\mathrm{pH}$ of fusion mutants within a few passages [51]. Improving virus growth in cell culture is important for producing high yields of virus for vaccines, and this enhanced growth is often associated with a broader $\mathrm{pH}$ range of virus-host fusion. Site-directed mutagenesis indicates that mutations in the HA2 fusion peptide [32,33,52] and the transmembrane domain [31] can stabilize HA to produce a virus that replicates to higher titers in cell culture. Taken together, these data suggest that cell adaptation correlates with decreased acid stability.

HA stability is only one known determinant of transmission; others include receptor binding, polymerase activity, and virus morphology [53]. Specific mutations in the receptor-binding site of HA can cause altered receptor preferences [54]. Although amino acids T212 and G78 lie outside the receptor-binding pocket, further studies are needed to determine their impact on receptor specificity and avidity, which may also contribute to the observed decrease in airborne transmission. Influenza viruses are pleiomorphic structures, with HA and NA packed closely but irregularly distributed on the surface of the virus particle [55-57]. NA is typically present in much smaller quantities that HA and it has been estimated that influenza virus particles have roughly $300 \mathrm{HA}$ and 20-40 NA proteins [56,58]. HA and NA have opposite functions and a fine balance is required for efficient virus replication, as HA binds to sialic-acid-containing receptors and NA removes sialic acid from host cells. Mutations that alter the NA enzymatic active site or stalk length have been linked to imbalance of the HA/NA relationship [59-61]. The pH of fusion phenotype for the rPerth mutant was mapped to both the HA G78D and T212I changes and X-31 UTRs flanking the NA segment (Figure 1A). This observation suggests an additional aspect of HA/NA balance whereby the noncoding regions of the NA gene segment can influence HA function. The viral gene segment UTRs are essential promoter elements required for initiation of viral replication and transcription. Viral gene expression requires interaction of the RNA-dependent RNA polymerase complex with both conserved sequence elements in the UTRs. Mutations in the UTR promoter region have been shown to modulate gene expression and downstream protein expression $[62,63]$. Therefore, changes in the NA UTR may alter the amount of NA synthesized during a viral infection and ultimately impact the amount of the protein present on a virion. Decreased incorporation of NA in virions has been observed to negatively impact NA activity and compensate for functional differences in HA $[64,65]$. The balance between HA and NA is critical for influenza virus fitness and a future avenue of research will be to determine the expression levels of mRNA and protein, and how these potential differences might be affected by different UTRs.

Supplementary Materials: The following are available online at https:/ / www.mdpi.com/article/10 .3390/v13050719/s1, Supplementary File 1: Sequence alignment of rPerth WT and mutant UTRs.

Author Contributions: V.L.S. and S.S.L. conceptualized the project and wrote the manuscript. V.L.S., J.D.B. and S.S.L. designed the experiments. V.L.S. and S.S.L. analyzed and interpreted the data. V.L.S., K.A.K., E.N., J.M.L. performed the experiments. M.M.M. and S.A.F. contributed to resources and sample preparation. All authors have read and agreed to the published version of the manuscript.

Funding: This research was funded by National Institute of Allergy and Infectious Diseases, grant numbers HHSN272201400007C (S.S.L.), 1R01AI139063-01A1 (S.S.L.) and R01AI127893 (J.D.B.). The APC was funded by National Institute of Allergy and Infectious Diseases, grant number 1R01AI139063-01A1.

Institutional Review Board Statement: The study approved by the Institutional Animal Care and Use Committee of the University of Pittsburgh (protocol code 19075697 and 2021-01-19). 
Informed Consent Statement: Not applicable.

Data Availability Statement: Data is contained within the article and supplementary material. All of the plasmid sequences are available as Snapgene files on FigShare at https:/ / figshare.com/projects / Cell_Culture_Adaptation_of_H3N2_Influenza_Virus_Impacts_Acid_Stability_and_Reduces_Airborne_ Transmission_in_Ferrets/112005 (accessed on 21 April 2021).

Acknowledgments: This work was supported by the National Institute of Allergy and Infectious Diseases (HHSN272201400007C, S.S.L.; 1R01AI139063-01A1, S.S.L.; R01AI127893, J.D.B.). Additional funding for SSL includes the American Lung Association Biomedical Research grant, and a New Initiative Award from the Charles E. Kaufman Foundation, a supporting organization of The Pittsburgh Foundation. S.A.F. and M.M.M. are funded through the Cystic Fibrosis Foundation Research Development Program to the University of Pittsburgh. We would like to thank the University of Pittsburgh DLAR technicians and veterinarians for their hard work and dedication as well as the members of Lakdawala lab for constructive discussions. J.D.B. is an Investigator of the Howard Hughes Medical Institute.

Conflicts of Interest: The authors declare that they have no conflict of interest.

\section{References}

1. Connor, R.J.; Kawaoka, Y.; Webster, R.G.; Paulson, J.C. Receptor specificity in human, avian, and equine H2 and H3 influenza virus isolates. Virology 1994, 205, 17-23. [CrossRef]

2. Shinya, K.; Ebina, M.; Yamada, S.; Ono, M.; Kasai, N.; Kawaoka, Y. Avian flu: Influenza virus receptors in the human airway. Nature 2006, 440, 435-436. [CrossRef]

3. van Riel, D.; Munster, V.J.; de Wit, E.; Rimmelzwaan, G.F.; Fouchier, R.A.; Osterhaus, A.D.; Kuiken, T. H5N1 Virus Attachment to Lower Respiratory Tract. Science 2006, 312, 399. [CrossRef]

4. Basak, S.; Tomana, M.; Compans, R.W. Sialic acid is incorporated into influenza hemagglutinin glycoproteins in the absence of viral neuraminidase. Virus Res. 1985, 2, 61-68. [CrossRef]

5. Palese, P.; Tobita, K.; Ueda, M.; Compans, R.W. Characterization of temperature sensitive influenza virus mutants defective in neuraminidase. Virology 1974, 61, 397-410. [CrossRef]

6. De Vries, E.; Du, W.; Guo, H.; de Haan, C.A.M. Influenza A Virus Hemagglutinin-Neuraminidase-Receptor Balance: Preserving Virus Motility. Trends Microbiol. 2020, 28, 57-67. [CrossRef] [PubMed]

7. Bottcher, E.; Matrosovich, T.; Beyerle, M.; Klenk, H.D.; Garten, W.; Matrosovich, M. Proteolytic activation of influenza viruses by serine proteases TMPRSS2 and HAT from human airway epithelium. J. Virol. 2006, 80, 9896-9898. [CrossRef] [PubMed]

8. Steinhauer, D.A. Role of hemagglutinin cleavage for the pathogenicity of influenza virus. Virology 1999, 258, 1-20. [CrossRef]

9. Skehel, J.J.; Wiley, D.C. Receptor binding and membrane fusion in virus entry: The influenza hemagglutinin. Annu. Rev. Biochem. 2000, 69, 531-569. [CrossRef] [PubMed]

10. Costello, D.A.; Whittaker, G.R.; Daniel, S. Variations in pH sensitivity, acid stability, and fusogenicity of three influenza virus H3 subtypes. J. Virol. 2015, 89, 350-360. [CrossRef] [PubMed]

11. Galloway, S.E.; Reed, M.L.; Russell, C.J.; Steinhauer, D.A. Influenza HA subtypes demonstrate divergent phenotypes for cleavage activation and $\mathrm{pH}$ of fusion: Implications for host range and adaptation. PLoS Pathog. 2013, 9, e1003151. [CrossRef]

12. Russier, M.; Yang, G.; Rehg, J.E.; Wong, S.S.; Mostafa, H.H.; Fabrizio, T.P.; Barman, S.; Krauss, S.; Webster, R.G.; Webby, R.J.; et al. Molecular requirements for a pandemic influenza virus: An acid-stable hemagglutinin protein. Proc. Natl. Acad. Sci. USA 2016, 113, 1636-1641. [CrossRef] [PubMed]

13. Gabbard, J.D.; Dlugolenski, D.; Van Riel, D.; Marshall, N.; Galloway, S.E.; Howerth, E.W.; Campbell, P.J.; Jones, C.; Johnson, S.; Byrd-Leotis, L.; et al. Novel H7N9 influenza virus shows low infectious dose, high growth rate, and efficient contact transmission in the guinea pig model. J. Virol. 2014, 88, 1502-1512. [CrossRef] [PubMed]

14. Hanson, A.; Imai, M.; Hatta, M.; McBride, R.; Imai, H.; Taft, A.; Zhong, G.; Watanabe, T.; Suzuki, Y.; Neumann, G.; et al. Identification of Stabilizing Mutations in an H5 Hemagglutinin Influenza Virus Protein. J. Virol. 2015, 90, 2981-2992. [CrossRef] [PubMed]

15. Herfst, S.; Mok, C.K.P.; van den Brand, J.M.A.; van der Vliet, S.; Rosu, M.E.; Spronken, M.I.; Yang, Z.; de Meulder, D.; Lexmond, P.; Bestebroer, T.M.; et al. Human Clade 2.3.4.4 A/H5N6 Influenza Virus Lacks Mammalian Adaptation Markers and Does Not Transmit via the Airborne Route between Ferrets. mSphere 2018, 3. [CrossRef] [PubMed]

16. Linster, M.; van Boheemen, S.; de Graaf, M.; Schrauwen, E.J.A.; Lexmond, P.; Manz, B.; Bestebroer, T.M.; Baumann, J.; van Riel, D.; Rimmelzwaan, G.F.; et al. Identification, characterization, and natural selection of mutations driving airborne transmission of A/H5N1 virus. Cell 2014, 157, 329-339. [CrossRef]

17. Richard, M.; Schrauwen, E.J.; de Graaf, M.; Bestebroer, T.M.; Spronken, M.I.; van Boheemen, S.; de Meulder, D.; Lexmond, P.; Linster, M.; Herfst, S.; et al. Limited airborne transmission of H7N9 influenza A virus between ferrets. Nature 2013, 501, 560-563. [CrossRef] [PubMed] 
18. Russier, M.; Yang, G.; Marinova-Petkova, A.; Vogel, P.; Kaplan, B.S.; Webby, R.J.; Russell, C.J. H1N1 influenza viruses varying widely in hemagglutinin stability transmit efficiently from swine to swine and to ferrets. PLoS Pathog. 2017, 13, e1006276. [CrossRef]

19. Watanabe, Y.; Arai, Y.; Daidoji, T.; Kawashita, N.; Ibrahim, M.S.; El-Gendy Eel, D.; Hiramatsu, H.; Kubota-Koketsu, R.; Takagi, T.; Murata, T.; et al. Characterization of $\mathrm{H} 5 \mathrm{~N} 1$ influenza virus variants with hemagglutinin mutations isolated from patients. $m B i o$ 2015, 6. [CrossRef]

20. Herfst, S.; Schrauwen, E.J.; Linster, M.; Chutinimitkul, S.; de Wit, E.; Munster, V.J.; Sorrell, E.M.; Bestebroer, T.M.; Burke, D.F.; Smith, D.J.; et al. Airborne transmission of influenza A/H5N1 virus between ferrets. Science 2012, 336, 1534-1541. [CrossRef]

21. Imai, M.; Watanabe, T.; Hatta, M.; Das, S.C.; Ozawa, M.; Shinya, K.; Zhong, G.; Hanson, A.; Katsura, H.; Watanabe, S.; et al. Experimental adaptation of an influenza H5 HA confers respiratory droplet transmission to a reassortant H5 HA/H1N1 virus in ferrets. Nature 2012, 486, 420-428. [CrossRef]

22. Russell, C.J.; Hu, M.; Okda, F.A. Influenza Hemagglutinin Protein Stability, Activation, and Pandemic Risk. Trends Microbiol. 2018, 26, 841-853. [CrossRef]

23. Lee, J.M.; Huddleston, J.; Doud, M.B.; Hooper, K.A.; Wu, N.C.; Bedford, T.; Bloom, J.D. Deep mutational scanning of hemagglutinin helps predict evolutionary fates of human H3N2 influenza variants. Proc. Natl. Acad. Sci. USA 2018, 115, E8276-E8285. [CrossRef] [PubMed]

24. Myerburg, M.M.; Harvey, P.R.; Heidrich, E.M.; Pilewski, J.M.; Butterworth, M.B. Acute regulation of the epithelial sodium channel in airway epithelia by proteases and trafficking. Am. J. Respir. Cell Mol. Biol. 2010, 43, 712-719. [CrossRef] [PubMed]

25. Reed, L.J.; Muench, H. A simple method of estimating fifty percent endpoints. Am. J. Hyg. 1938, $27,493-497$.

26. Hoffmann, E.; Neumann, G.; Kawaoka, Y.; Hobom, G.; Webster, R.G. A DNA transfection system for generation of influenza A virus from eight plasmids. Proc. Natl. Acad. Sci. USA 2000, 97, 6108-6113. [CrossRef] [PubMed]

27. Lakdawala, S.S.; Lamirande, E.W.; Suguitan, A.L., Jr.; Wang, W.; Santos, C.P.; Vogel, L.; Matsuoka, Y.; Lindsley, W.G.; Jin, H.; Subbarao, K. Eurasian-origin gene segments contribute to the transmissibility, aerosol release, and morphology of the 2009 pandemic H1N1 influenza virus. PLoS Pathog. 2011, 7, e1002443. [CrossRef]

28. Lakdawala, S.S.; Jayaraman, A.; Halpin, R.A.; Lamirande, E.W.; Shih, A.R.; Stockwell, T.B.; Lin, X.; Simenauer, A.; Hanson, C.T.; Vogel, L.; et al. The soft palate is an important site of adaptation for transmissible influenza viruses. Nature 2015, 526, 122-125. [CrossRef]

29. Le Sage, V.; Jones, J.E.; Kormuth, K.A.; Fitzsimmons, W.J.; Nturibi, E.; Padovani, G.H.; Arevalo, C.P.; French, A.J.; Avery, A.J.; Manivanh, R.; et al. Pre-existing heterosubtypic immunity provides a barrier to airborne transmission of influenza viruses. PLoS Pathog. 2021, 17, e1009273. [CrossRef]

30. Nturibi, E.; Bhagwat, A.R.; Coburn, S.; Myerburg, M.M.; Lakdawala, S.S. Intracellular Colocalization of Influenza Viral RNA and Rab11A Is Dependent upon Microtubule Filaments. J. Virol. 2017, 91. [CrossRef]

31. Kawahara, M.; Wada, T.; Momose, F.; Nobusawa, E.; Morikawa, Y. Cell-Based Influenza A/H1N1pdm09 Vaccine Viruses Containing Chimeric Hemagglutinin with Improved Membrane Fusion Ability. Vaccines 2020, 8, 458. [CrossRef]

32. Murakami, S.; Horimoto, T.; Ito, M.; Takano, R.; Katsura, H.; Shimojima, M.; Kawaoka, Y. Enhanced growth of influenza vaccine seed viruses in vero cells mediated by broadening the optimal $\mathrm{pH}$ range for virus membrane fusion. J. Virol. 2012, 86, 1405-1410. [CrossRef]

33. Yang, H.; Chang, J.C.; Guo, Z.; Carney, P.J.; Shore, D.A.; Donis, R.O.; Cox, N.J.; Villanueva, J.M.; Klimov, A.I.; Stevens, J. Structural stability of influenza A(H1N1)pdm09 virus hemagglutinins. J. Virol. 2014, 88, 4828-4838. [CrossRef] [PubMed]

34. Zaraket, H.; Bridges, O.A.; Duan, S.; Baranovich, T.; Yoon, S.W.; Reed, M.L.; Salomon, R.; Webby, R.J.; Webster, R.G.; Russell, C.J. Increased acid stability of the hemagglutinin protein enhances $\mathrm{H} 5 \mathrm{~N} 1$ influenza virus growth in the upper respiratory tract but is insufficient for transmission in ferrets. J. Virol. 2013, 87, 9911-9922. [CrossRef] [PubMed]

35. Zaraket, H.; Bridges, O.A.; Russell, C.J. The $\mathrm{pH}$ of activation of the hemagglutinin protein regulates H5N1 influenza virus replication and pathogenesis in mice. J. Virol. 2013, 87, 4826-4834. [CrossRef] [PubMed]

36. Hu, M.; Yang, G.; DeBeauchamp, J.; Crumpton, J.C.; Kim, H.; Li, L.; Wan, X.F.; Kercher, L.; Bowman, A.S.; Webster, R.G.; et al. HA stabilization promotes replication and transmission of swine H1N1 gamma influenza viruses in ferrets. Elife 2020, 9 . [CrossRef] [PubMed]

37. Belser, J.A.; Katz, J.M.; Tumpey, T.M. The ferret as a model organism to study influenza A virus infection. Dis. Model. Mech. 2011, 4, 575-579. [CrossRef]

38. Shelton, H.; Roberts, K.L.; Molesti, E.; Temperton, N.; Barclay, W.S. Mutations in haemagglutinin that affect receptor binding and $\mathrm{pH}$ stability increase replication of a PR8 influenza virus with H5 HA in the upper respiratory tract of ferrets and may contribute to transmissibility. J. Gen. Virol. 2013, 94, 1220-1229. [CrossRef]

39. Reed, M.L.; Bridges, O.A.; Seiler, P.; Kim, J.K.; Yen, H.L.; Salomon, R.; Govorkova, E.A.; Webster, R.G.; Russell, C.J. The pH of activation of the hemagglutinin protein regulates $\mathrm{H} 5 \mathrm{~N} 1$ influenza virus pathogenicity and transmissibility in ducks. J. Virol. 2010, 84, 1527-1535. [CrossRef]

40. Richard, M.; Herfst, S.; van den Brand, J.M.A.; de Meulder, D.; Lexmond, P.; Bestebroer, T.M.; Fouchier, R.A.M. Mutations Driving Airborne Transmission of A/H5N1 Virus in Mammals Cause Substantial Attenuation in Chickens only when combined. Sci. Rep. 2017, 7, 7187. [CrossRef] 
41. Hartley, C.A.; Reading, P.C.; Ward, A.C.; Anders, E.M. Changes in the hemagglutinin molecule of influenza type A (H3N2) virus associated with increased virulence for mice. Arch. Virol. 1997, 142, 75-88. [CrossRef] [PubMed]

42. Keleta, L.; Ibricevic, A.; Bovin, N.V.; Brody, S.L.; Brown, E.G. Experimental evolution of human influenza virus H3 hemagglutinin in the mouse lung identifies adaptive regions in HA1 and HA2. J. Virol. 2008, 82, 11599-11608. [CrossRef] [PubMed]

43. Koerner, I.; Matrosovich, M.N.; Haller, O.; Staeheli, P.; Kochs, G. Altered receptor specificity and fusion activity of the haemagglutinin contribute to high virulence of a mouse-adapted influenza A virus. J. Gen. Virol. 2012, 93, 970-979. [CrossRef] [PubMed]

44. Klein, E.Y.; Blumenkrantz, D.; Serohijos, A.; Shakhnovich, E.; Choi, J.M.; Rodrigues, J.V.; Smith, B.D.; Lane, A.P.; Feldman, A.; Pekosz A. Stability of the Influenza Virus Hemagglutinin Protein Correlates with Evolutionary Dynamics. mSphere 2018, 3. [CrossRef]

45. Labadie, T.; Batejat, C.; Manuguerra, J.C.; Leclercq, I. Influenza Virus Segment Composition Influences Viral Stability in the Environment. Front. Microbiol. 2018, 9, 1496. [CrossRef] [PubMed]

46. Poulson, R.L.; Tompkins, S.M.; Berghaus, R.D.; Brown, J.D.; Stallknecht, D.E. Environmental Stability of Swine and Human Pandemic Influenza Viruses in Water under Variable Conditions of Temperature, Salinity, and pH. Appl. Environ. Microbiol. 2016, 82, 3721-3726. [CrossRef] [PubMed]

47. Russier, M.; Yang, G.; Briard, B.; Meliopoulos, V.; Cherry, S.; Kanneganti, T.D.; Schultz-Cherry, S.; Vogel, P.; Russell, C.J. Hemagglutinin Stability Regulates H1N1 Influenza Virus Replication and Pathogenicity in Mice by Modulating Type I Interferon Responses in Dendritic Cells. J. Virol. 2020, 94. [CrossRef] [PubMed]

48. Singanayagam, A.; Zambon, M.; Barclay, W.S. Influenza Virus with Increased pH of Hemagglutinin Activation Has Improved Replication in Cell Culture but at the Cost of Infectivity in Human Airway Epithelium. J. Virol. 2019, 93. [CrossRef] [PubMed]

49. Marvin, S.A.; Russier, M.; Huerta, C.T.; Russell, C.J.; Schultz-Cherry, S. Influenza Virus Overcomes Cellular Blocks To Productively Replicate, Impacting Macrophage Function. J. Virol. 2017, 91. [CrossRef]

50. Gerlach, T.; Hensen, L.; Matrosovich, T.; Bergmann, J.; Winkler, M.; Peteranderl, C.; Klenk, H.D.; Weber, F.; Herold, S.; Pohlmann, S.; et al. pH Optimum of Hemagglutinin-Mediated Membrane Fusion Determines Sensitivity of Influenza A Viruses to the Interferon-Induced Antiviral State and IFITMs. J. Virol. 2017, 91. [CrossRef]

51. Lin, Y.P.; Wharton, S.A.; Martin, J.; Skehel, J.J.; Wiley, D.C.; Steinhauer, D.A. Adaptation of egg-grown and transfectant influenza viruses for growth in mammalian cells: Selection of hemagglutinin mutants with elevated $\mathrm{pH}$ of membrane fusion. Virology 1997, 233, 402-410. [CrossRef]

52. Cotter, C.R.; Jin, H.; Chen, Z. A single amino acid in the stalk region of the H1N1pdm influenza virus HA protein affects viral fusion, stability and infectivity. PLoS Pathog. 2014, 10, e1003831. [CrossRef]

53. Long, J.S.; Mistry, B.; Haslam, S.M.; Barclay, W.S. Host and viral determinants of influenza A virus species specificity. Nat. Rev. Microbiol. 2019, 17, 67-81. [CrossRef]

54. de Graaf, M.; Fouchier, R.A. Role of receptor binding specificity in influenza A virus transmission and pathogenesis. EMBO J. 2014, 33, 823-841. [CrossRef] [PubMed]

55. Calder, L.J.; Wasilewski, S.; Berriman, J.A.; Rosenthal, P.B. Structural organization of a filamentous influenza A virus. Proc. Natl. Acad. Sci. USA 2010, 107, 10685-10690. [CrossRef]

56. Harris, A.; Cardone, G.; Winkler, D.C.; Heymann, J.B.; Brecher, M.; White, J.M.; Steven, A.C. Influenza virus pleiomorphy characterized by cryoelectron tomography. Proc. Natl. Acad. Sci. USA 2006, 103, 19123-19127. [CrossRef]

57. Leser, G.P.; Lamb, R.A. Lateral Organization of Influenza Virus Proteins in the Budozone Region of the Plasma Membrane. J. Virol. 2017, 91. [CrossRef] [PubMed]

58. Hutchinson, E.C.; Charles, P.D.; Hester, S.S.; Thomas, B.; Trudgian, D.; Martinez-Alonso, M.; Fodor, E. Conserved and host-specific features of influenza virion architecture. Nat. Commun. 2014, 5, 4816. [CrossRef] [PubMed]

59. McKimm-Breschkin, J.L.; Barrett, S.; McKenzie-Kludas, C.; McAuley, J.; Streltsov, V.A.; Withers, S.G. Passaging of an influenza A(H1N1)pdm09 virus in a difluoro sialic acid inhibitor selects for a novel, but unfit I106M neuraminidase mutant. Antivir. Res. 2019, 169, 104542. [CrossRef] [PubMed]

60. Mitnaul, L.J.; Matrosovich, M.N.; Castrucci, M.R.; Tuzikov, A.B.; Bovin, N.V.; Kobasa, D.; Kawaoka, Y. Balanced hemagglutinin and neuraminidase activities are critical for efficient replication of influenza A virus. J. Virol. 2000, 74, 6015-6020. [CrossRef]

61. Romero-Beltran, L.; Baker, S.F.; Puerto-Solis, M.; Gonzalez-Losa, R.; Conde-Ferraez, L.; Alvarez-Sanchez, L.C.; Martinez-Sobrido, L.; Ayora-Talavera, G. Mutations at highly conserved residues in influenza A(H1N1)pdm09 virus affect neuraminidase activity. Virus Res. 2016, 225, 1-9. [CrossRef]

62. Maamary, J.; Pica, N.; Belicha-Villanueva, A.; Chou, Y.Y.; Krammer, F.; Gao, Q.; Garcia-Sastre, A.; Palese, P. Attenuated influenza virus construct with enhanced hemagglutinin protein expression. J. Virol. 2012, 86, 5782-5790. [CrossRef]

63. Neumann, G.; Hobom, G. Mutational analysis of influenza virus promoter elements in vivo. J. Gen. Virol. 1995, 76, 1709-1717. [CrossRef] [PubMed]

64. Das, S.R.; Hensley, S.E.; Ince, W.L.; Brooke, C.B.; Subba, A.; Delboy, M.G.; Russ, G.; Gibbs, J.S.; Bennink, J.R.; Yewdell, J.W. Defining influenza A virus hemagglutinin antigenic drift by sequential monoclonal antibody selection. Cell Host Microbe 2013, 13, 314-323. [CrossRef] [PubMed]

65. Hensley, S.E.; Das, S.R.; Gibbs, J.S.; Bailey, A.L.; Schmidt, L.M.; Bennink, J.R.; Yewdell, J.W. Influenza A virus hemagglutinin antibody escape promotes neuraminidase antigenic variation and drug resistance. PLoS ONE 2011, 6, e15190. [CrossRef] [PubMed] 